\title{
A New High Order Accurate Shock Capture Method with Wave Booster
}

\author{
Yanwen $\mathrm{Ma}^{\mathrm{a}}$, Dexun $\mathrm{Fu}^{\mathrm{a}}$, and Xinliang $\mathrm{Li}^{\mathrm{a}}$ \\ ${ }^{a}$ Institute of Mechanics, Chinese Academy of Sciences \\ ( fudx@Inm.imech.ac.cn)
}

\begin{abstract}
A new kind of shock capturing method is developed. Before applying the high order accurate traditional scheme which is called as base scheme in this paper the fluid parameters are preconditioned in order to control the group velocity. The newly constructed scheme is high order accurate, simple, has high resolution of the shock, and less computer time consumed.
\end{abstract}

Keywords: shock capturing, high order accurate scheme, group velocity control.

PACS: 47.11。Bc

\section{INTRODUCTION}

Direct numerical simulations require all the relevant scales to be properly represented in the numerical model. For solving such kind of problems high order accurate methods are preferred ${ }^{[1-3]}$.In the compressible flow fields there may exist the shocks which are discontinuous surfaces for the inviscid flow and surfaces with large cross gradient of the fluid parameters for the viscous flows. Many efficient high order accurate schemes are developed for shock capturing, and they are used to solve a lot of practical engineering problems ${ }^{[4-6]}$. In this paper a new kind of high order accurate schemes with high resolution of the shock is developed. The solution process consists of two steps. In the first step the fluid parameters at time $t$ is preconditioned by so called wave booster. In the second step we put the preconditioned fluid parameters into a base scheme, and obtain a finally difference approximation. It is required that the finally obtained scheme is FST/MXD behind the shocks and SLW in front the shocks ${ }^{[5]}$. The constructed scheme by this way is high order accurate and can capture the shocks well.

\section{GROUP VELOCITY}

Consider the following model equation and its semi-discrete approximation

$$
\begin{gathered}
\frac{\partial U}{\partial t}=L(U)=-\frac{\partial f}{\partial x}, \quad f=c U, \quad c=\text { const } \\
\frac{\partial U_{j}}{\partial t}=L_{h}\left(U_{j}\right)=-\frac{F_{j}}{\Delta x}
\end{gathered}
$$

where $F_{j} / \Delta x$ is an approximation of the first derivative $\partial f / \partial x$, for example, $F_{j}(f)=\left(f_{j+1}-f_{j-1}\right) / 2$. Putting a single Fourier component $f_{j}=c \exp \left(i k_{j}\right)$ into (2) we have $F_{j}(f)=c\left[k_{r}(\alpha)+i k_{i}(\alpha)\right]$ where $i=\sqrt{-1}$ and $\alpha=k \Delta x \cdot k_{r}(\alpha)$ corresponds to the dissipation rate and $k_{i}(\alpha)$ corresponds the to the modified wave number. For the second order central deference we have $k_{r}(\alpha)=0$, and $k_{i}(\alpha)=\sin (\alpha)$. In Ref.[5] the 
group velocity is defined as $D(\alpha)=d k_{i}(\alpha) / d \alpha$, and the difference schemes are divided into three classes: slow(SLW), fast( FST) and mixed(MXD). We have $D(\alpha) \leq 1$ for the scheme from SLW, $D(\alpha) \geq 1$ for the scheme from FST, and $D\left(\alpha<\alpha_{0}\right) \geq 1$ and $D\left(\alpha>\alpha_{0}\right) \leq 1$ for the scheme from MXD where $\alpha_{0}$ depends on particular scheme and $0<\alpha_{0}<\pi$.

It is stated in Ref.[5] that the reason of oscillation production in the numerical solutions is due to non-uniform group velocity of wave components in the numerical solutions. The oscillations in numerical solutions are produced behind the shock(upstream of the shock) for the SLW schemes, in front of the shock(downstream of the shock) for the FST schemes. For the scheme from MXD the oscillations are produced in front of the shock for the lower and moderate wave components, and they are produced behind the shock for the high and super high wave components.

For improvements of the shock resolution the scheme should be reconstructed so that the scheme exhibits FST/MXD property behind the shock, and SLW property in front of the shock.

\section{SCHEME CONSTRUCTION}

A new way to construct the shock capturing schemes is presented. The solution process consists of two sub-processes. In the first sub-process the fluid parameters at time step $\mathrm{n}$ are preconditioned by a so called wave booster(WB)with specially selected control parameter. The second sub-process is the process of discretization of the fluid parameters with the base difference from class SLW after wave boosting. The finally obtained scheme exhibits property of the scheme from FST/MXD behind the shock, and property of the scheme from SLW in front of the shock.

We chose the $2 \mathrm{k}+1$ order accurate weakly upwind biased schemes as the base schemes which can be written for the case $\mathrm{c}>0$ as follows

$$
\begin{gathered}
F\left(f_{j}^{n}\right)=\sum_{l=-k}^{k} a_{l}\left(f_{j+l}^{n}-f_{j+l-1}^{n}\right)=h_{j+1 / 2}-h_{j-1 / 2} \\
\sum_{l} a_{l}=1 \\
\sum_{l=-k}^{k} a_{l}\left[l^{m}-(l-1)^{m}\right]=0, \quad m=2, \cdots, 2 k+1
\end{gathered}
$$

The coefficients $a_{l}$ can be obtained by solving the linear system of algebraic equations (4) and (5) according the accuracy requirement.

For the wave booster(WB) the following conditions need to be satisfied:

a) WB can turn the group velocity into direction what we need for improvement of shock resolution;

b) the accuracy of the schemes is not changed;

c) the booster is simple.

In this paper the following WB operator is suggested

$$
\hat{f}_{j}=W B^{(2 k+1)}\left(f_{j}\right)=f_{j}-\sigma^{+} \frac{(-1)^{k+1}}{2^{k}} \delta_{x}^{2 k} f_{j}
$$

where $\sigma_{j}^{+} \approx O(\Delta x)$ in the smooth region. Putting (6) into (3) we obtain

$$
F\left(\hat{f}_{j}^{n}\right)=\sum_{l=-k}^{k} a_{l}\left(\hat{f}_{j+l}^{n}-\hat{f}_{j+l-1}^{n}\right)
$$

Putting $f_{j}=\exp \left[i k x_{j}\right\rfloor$ in to (3) and (6), and $\hat{f}_{j}=\hat{T}(\alpha) \exp \left[i k x_{j}\right]$ into (7), we obtain

$$
\begin{aligned}
F\left(f_{j}\right)=K e(\alpha) \exp \left[i k x_{j}\right\rfloor, & F\left(\hat{f}_{j}\right)=\hat{K} e(\alpha) \exp \left[i k x_{j}\right] \\
\operatorname{Ke}(\alpha)=\operatorname{Kr}(\alpha)+i K i(\alpha) & \hat{K} e(\alpha)=\hat{T} \bullet K e=\hat{K} r(\alpha)+i \hat{K} i(\alpha)
\end{aligned}
$$




$$
\begin{array}{ccc}
\hat{K} r(\alpha)= & K r(\alpha) \bullet \hat{T}(\alpha), & \hat{K} i(\alpha)=K i(\alpha) \bullet \hat{T}(\alpha) \\
\hat{T}(\alpha)=1+\sigma^{+}[1-\cos (\alpha)]^{k} & \sigma^{+}>0
\end{array}
$$

From (8) and (9) we see that the wave booster is some kind of an amplifier.

\section{ACCURACY AND BEHAVIOR ANALYSIS}

The above listed base schemes are dissipative, and belong to the class SLW. After wave booster treatment with selected parameter $\sigma$ we can turn the SLW scheme into MXD scheme. We use two way to analyze the accuracy and behavior of the numerical solutions, the Taylor series expansion, and the Fourier analysis. follows

The modified equation for the case of third order accurate base scheme $(c>0)$ after wave booster treatment is as

$$
\frac{\partial U_{j}}{\partial t}+\frac{\partial f_{j}}{\partial x}=\sigma^{+} \Delta x^{2} \frac{\partial^{3} f_{j}}{\partial x^{3}}-\frac{1}{12} \Delta x^{3} \frac{\partial^{4} f_{j}}{\partial x^{4}}+\cdots
$$

Consider the leading terms on the right hand side of (10). The term with fourth derivative is produced from the original third order accurate scheme $(\mathrm{c}>0)$, and it gives the positive dissipation. The term with third derivative is newly produced by wave boosting, and it does not change the accuracy of the scheme with $\sigma^{+} \approx \Delta x$. We see that the introduced term by wave booster gives positive dispersion, and the amount of dispersion, and the group velocity can be controlled by the parameter $\sigma^{+}$.The above analysis can be done for the higher order accurate base schemes with higher order accurate wave booster.

The accuracy and the behavior of the numerical solutions also can be discussed by the Fourier analysis. Putting the single Fourier component into the wave booster treated scheme we can obtain $\hat{K} i(\alpha), \hat{K} r(\alpha)$.and the group velocity $d[\hat{K} i(\alpha)] / d \alpha$. In Fig.1 are shown the variations of the group velocity $d[\hat{K} i(\alpha)] / d \alpha$ and the dissipation rate $\hat{K} r(\alpha)$ as function $\alpha$ for the $7^{\text {th }}$ order accurate base scheme with different parameter $\sigma^{+}$.

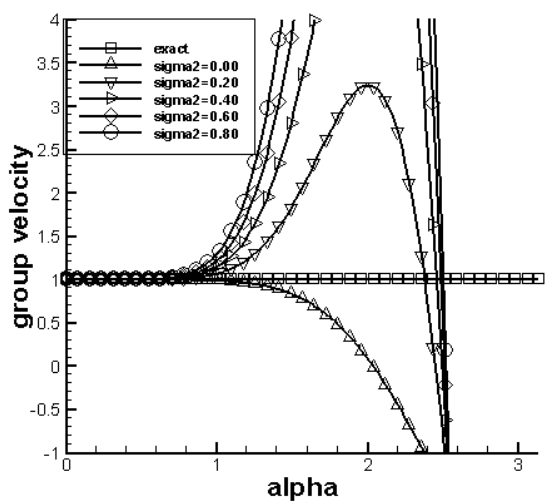

FIGURE 1. (a) group velocity for $\mathrm{k}=3$ with different $\sigma$

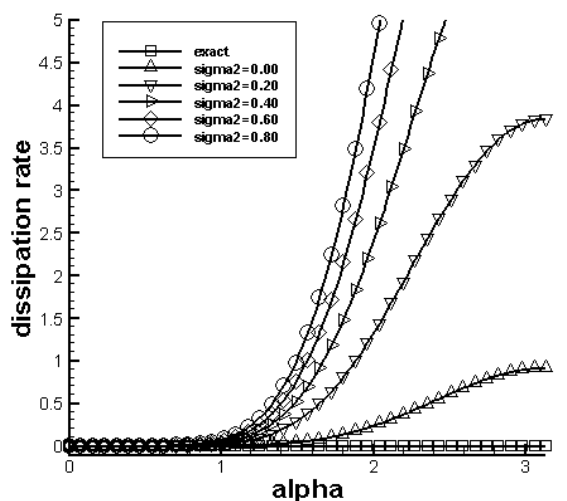

FIGURE 1. (b) dissipation rate $\mathrm{k}=3$ with different $\sigma$

From Fig. 1 we can also see that the scheme is dissipative and belongs to the class SLW for the case of $\sigma^{+}=0$. With increasing the parameter $\sigma^{+}$the booster turns the SLW scheme into the scheme which belong to the class MXD. From figures it also can be seen that the dissipation rate is increased with increasing the parameter $\sigma^{+}$.

\section{DISCRETIZATION OF EULER EQUATIONS}

Consider the one dimensional Euler equations in vector form

$$
\frac{\partial}{\partial t} \boldsymbol{U}+\frac{\partial}{\partial x} \boldsymbol{f}=\mathbf{0}
$$


where

$$
\begin{array}{ll}
\boldsymbol{U}=[\rho, \rho u, E]^{\mathrm{T}}, & \boldsymbol{f}=\left[\rho u, \rho u^{2}+p, u(E+p)\right]^{\mathrm{T}} \\
\boldsymbol{U}^{(0)}=[\rho, u, T]^{\mathrm{T}}, & E=\rho\left(C v T+V^{2} / 2\right)
\end{array}
$$

With Steger-Warming flux vector splitting we have the following wave treated semi-discrete difference approximation

$$
\begin{gathered}
\frac{\partial \boldsymbol{U}_{j}}{\partial t}+\frac{F^{+}\left(\hat{U}_{j}^{(0),+}\right)}{\Delta x}+\frac{F^{-}\left(\hat{U}_{j}^{(0),-}\right)}{\Delta x}=\mathbf{0} \\
\hat{\boldsymbol{U}}_{j}^{(0), \pm}=W B^{(2 k+1)}\left(\boldsymbol{U}_{j}^{(0)}\right)=\boldsymbol{U}_{j}^{(0)}-\sigma_{j}^{ \pm} \frac{(-1)^{k+1}}{2^{k}} \delta_{x}^{2 k} \boldsymbol{U}_{j}^{(0)}
\end{gathered}
$$

The control parameter function $\sigma_{j}^{ \pm}$is defined in this paper as follows

$$
\begin{aligned}
& \sigma_{j}^{ \pm}=\varepsilon_{j}\left\{\left[1 \pm S S\left(p_{j}\right)\right] \sigma_{u}^{ \pm} / 2+\left[1 \mp S S\left(p_{j}\right)\right] \sigma_{d}^{ \pm} / 2\right\} \\
& \quad S S\left(p_{j}\right)=\operatorname{sign}\left(\delta_{x}^{0} p_{j}, \delta_{x}^{2} p_{j}\right) \\
& \varepsilon_{j}=\frac{\left|\delta_{x}^{+} p_{j}\right|+\left|\delta_{x}^{-} p_{j}\right|}{p_{j+1}+2 p_{j}+p_{j-1}}, \quad \delta_{x}^{0}=\frac{1}{2}\left(\delta_{x}^{+}+\delta_{x}^{-}\right), \quad \delta_{x}^{ \pm} f_{j}=\mp\left(f_{i}-f_{j \pm 1}\right)
\end{aligned}
$$

In present computation $\sigma_{u}^{ \pm}=1, \sigma_{d}^{ \pm}=0$ is used.

\section{NUMERICAL EXAMPLES}

The above presented methods with order of accuracy $2 \mathrm{k}+1(\mathrm{k}=1,2,3,4)$ are used to solve some model problems such as 1-D and 2-D Sod model problems, 2-D Riemann problems, and sound generation by shock-vortex interaction. Only part of the computed results are shown here.

Sod model problem. A tube extends from $\mathrm{x}=0$ to $\mathrm{x}=1$ is divided by 100 equal intervals. with $p=\rho=1$, and $u=0$ in the subdomain [0,0.5], and $p=0.1, \rho=0.125, u=0$ in the subdomain $(0.5,1.0]$ where. $p, \rho$ and $\mathrm{u}$ are the pressure, density and velocity, respectively. The one-dimensional Euler equations are discretized by the $7^{\text {th }}$ order accurate base scheme with the wave booster $(6)(\mathrm{k}=3)$. Fig.2 shows the obtained density and pressure distribution.

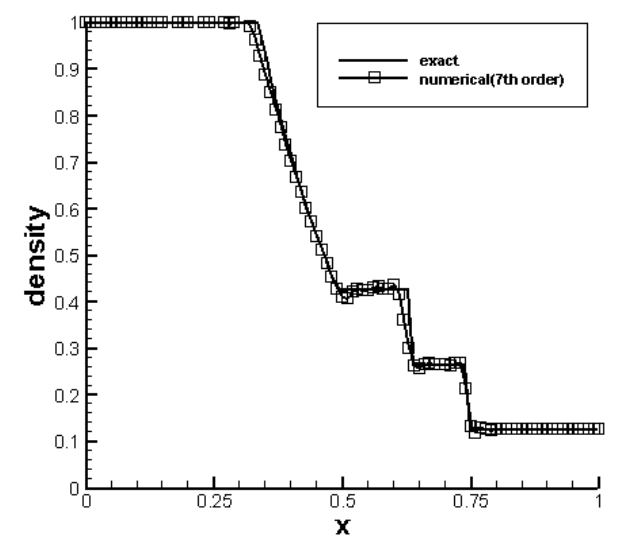

FIGURE 2. (a) Density distribution with $7^{\text {th }}$ order scheme

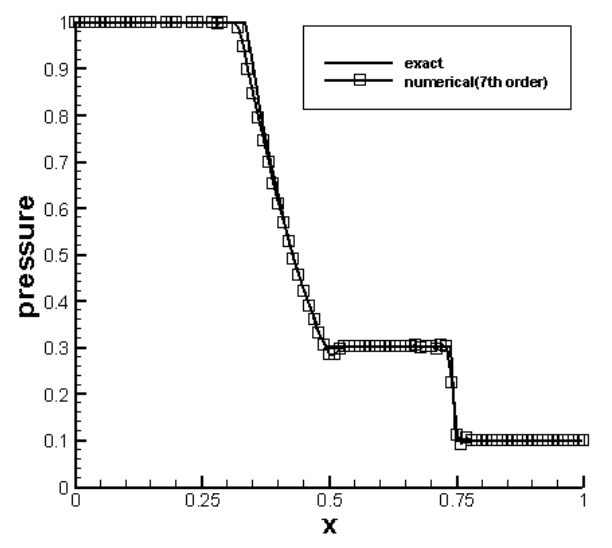

FIGURE 2. (b) pressure distribution with $7^{\text {th }}$ order scheme

2-D Riemann problem The second example is a two-dimensional Riemann problem [7] .In computation $N X \times N Y=401 \times 401$ grid points are used. This problem was computed by the $7^{\text {th }}$ order accurate 
base scheme with wave booster $\mathrm{k}=3$. The contours of density and pressure at $\mathrm{t}=0.52$ are given in Fig. 3 The shocks and the slip lines can be seen clearly. In the figures down-left we also can see the smaller structures.

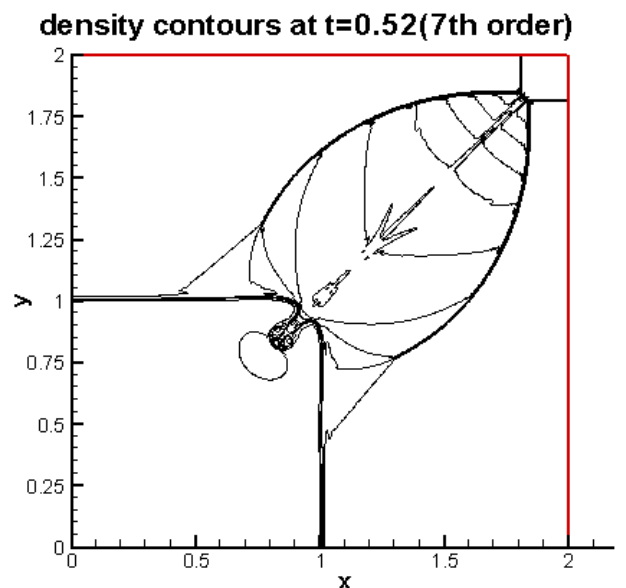

FIGURE 3. (a) Density distribution with $7^{\text {th }}$ order upwind scheme

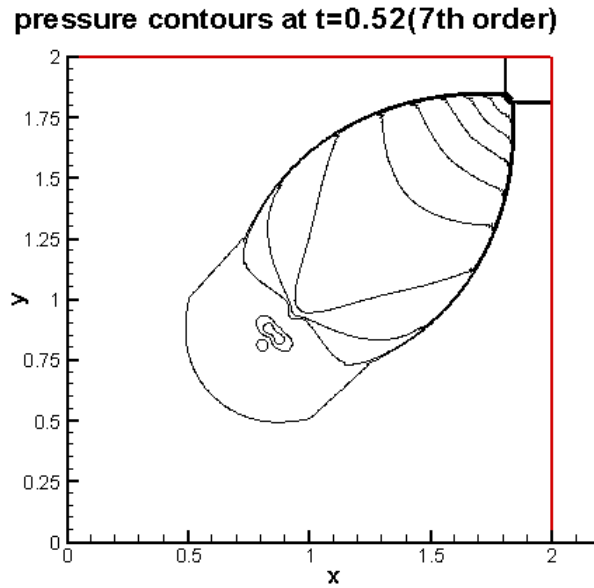

FIGURE 3. (b) pressure distribution with $7^{\text {th }}$ order upwind scheme

\section{SUMMARY}

A new high order accurate scheme with wave booster is developed, and the accuracy and behavior of the numerical solution are analyzed The developed scheme is simple, less computer time consumed, and can capture the shocks well. The scheme is used to solve practical problems, and obtained results are satisfactory.

\section{ACKNOWLEDGEMENTS}

This work was supported by National Natural Science Foundation of China (Grant Nos. 10632050, 10872205).

\section{REFERENCES}

1. Lele, SK, Journal of computational physics,1992;103:16-42.

2. Fu Dexun and Ma Yanwen, Computational Fluid Dynamics Review 1995, John Wiley \& Sons, ed. By M.Hafez and K. Oshima, 1995;234-250.

3. Ma Yanwen and Fu Dexun, Computational Fluid Dynamics Journal, 1996, 5(2): 259—276, 10.

4. C-W. Shu, Lecture notes in computational science and engineering(9), Springer-Verlag, 1999.

5 Fu Dexun and Ma Yanwen, Journal of computational physics, 1997; 134:1-15.

6 Yee H, Sjogreen B, Proc. $3^{\text {rd }}$ international conference on computational dynamics, Toronto, 2004;407-414.

7 Brio M,et al, Journal of computational physics, 2001;167:177-195. 